\title{
Viewing Pictures Triggers Rapid Morphological Enlargement in the Human Visual Cortex
}

\author{
Kristoffer N. T. Månsson (1) 1,2,3,*, Diana S. Cortes ${ }^{1}$, Amir Manzouri ${ }^{1}$, \\ Tie-Qiang Li ${ }^{4,5}$, Stephan Hau ${ }^{1}$ and Håkan Fischer ${ }^{1}$
}

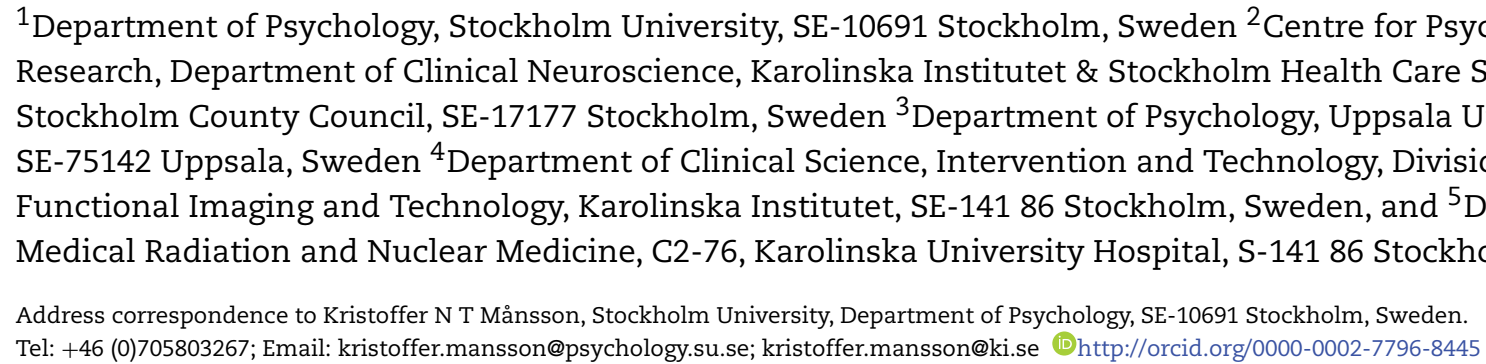

\begin{abstract}
Measuring brain morphology with non-invasive structural magnetic resonance imaging is common practice, and can be used to investigate neuroplasticity. Brain morphology changes have been reported over the course of weeks, days, and hours in both animals and humans. If such short-term changes occur even faster, rapid morphological changes while being scanned could have important implications. In a randomized within-subject study on 47 healthy individuals, two high-resolution T1-weighted anatomical images were acquired (á $263 \mathrm{~s}$ ) per individual. The images were acquired during passive viewing of pictures or a fixation cross. Two common pipelines for analyzing brain images were used: voxel-based morphometry on gray matter (GM) volume and surface-based cortical thickness. We found that the measures of both GM volume and cortical thickness showed increases in the visual cortex while viewing pictures relative to a fixation cross. The increase was distributed across the two hemispheres and significant at a corrected level. Thus, brain morphology enlargements were detected in less than $263 \mathrm{~s}$. Neuroplasticity is a far more dynamic process than previously shown, suggesting that individuals' current mental state affects indices of brain morphology. This needs to be taken into account in future morphology studies and in everyday clinical practice.
\end{abstract}

Key words: gray matter volume, cortical thickness, neuroplasticity, voxel-based morphometry, surface-based thickness

\section{Introduction}

T1-weighted magnetic resonance imaging (MRI) is extensively used in both structural brain imaging research and clinical practice. Non-invasive MRI and T1-weighted images represent a macro-level assessment of brain morphology (Tardif et al. 2016; Wenger et al. 2017a), for example, different tissues, cell types, synapses, and dendritic spines in the cellular milieu. Morphological assessments are routinely used, for example, to assess brain atrophy in normal aging (Nyberg et al. 2010) neurodegenerative disease (Benzinger et al. 2013), and in psychiatric disorders (Hoogman et al. 2017). Further, a plethora of human neuroplasticity studies suggest remarkable plastic volumetric alterations induced by motor training (Wenger et al. 2017b), 
physical activity (Erickson et al. 2011), and by pharmacological agents (Tost et al. 2010). Substantial attention has been directed toward a number of studies on human brain plasticity. For instance, increased gray matter (GM) volume in London taxi drivers (Maguire et al. 2000) or volumetric changes induced by practicing mindfulness (Hölzel et al. 2011) or juggling (Draganski et al. 2004). Such structural changes in humans are typically found after several weeks, but there is also suggestive evidence of neuroplasticity already after days (Kwok et al. 2011) or less. Tost et al. (2010) reported changes after less than $2 \mathrm{~h}$, both in striatal GM volume and brain response coupling in individuals undergoing an acute dopamine D2 antagonists challenge. Thus, brain morphology is dynamic and alters rapidly by both internal and external environmental influences.

Studies demonstrating changes in less than a few hours raise the question on how fast brain morphology could alter, and if rapid changes at scanning are possible to capture with noninvasive MRI techniques. In contrast to previous studies measuring changes between time points, an alternative approach could be to measure brain morphology alterations more directly and during anatomical scanning. Therefore, to test if the brain's morphology alters already at time of assessment, we conducted a simple experimental manipulation while acquiring T1-weighted anatomical images. In particular, using a randomized balanced within-subject design, 47 healthy participants underwent T1weighted MRI image acquisition while passively viewing complex arousing pictures or a fixation cross.

\section{Materials and Methods}

\section{Experimental Design}

This study used a randomized within-subject design. All participants underwent T1-weighted MRI measurements under the following two conditions: 1) a task condition while viewing arousing complex pictures, and B) a baseline condition while focusing on a fixation cross. The order in which the conditions were presented was randomized across participants. The second T1-weighted measurement was completed within the same scanning session and there was no stimuli or task in between the two assessments.

The experimental task was to passively view images from the International Affective Picture System (IAPS). The selected IAPS images were either of negative or positive valence, mixed and highly arousing (Lang 2005) to foster attention and avoid habituation (Fischer et al. 2003). Each picture was displayed for 8 $\mathrm{s}$ and the pictures were presented in a random order. There were 15 different pictures and in total 33 trials presented during the acquisition. In contrast, during the baseline condition, images were acquired while participants were focusing on a fixation cross presented in the center of the screen. Participants viewed the visual stimulus via a tilted mirror attached to the head coil.

\section{Participants}

Individuals were recruited via social media ads or emails to university students. Fifty-two subjects were recruited and 47 healthy individuals remained for the final analysis: $28.0 \pm 7.1$ mean years, 74.5\% (35/47) females, 95.7\% (45/47) right-handed, with a mean body mass index of $21.7 \pm 2.4$. Participants rated their health to be good (as determined by the self-rated health scale, SRH-7; mean \pm SD 5.8 \pm 0.9 , where seven represented excellent health). All participants fulfilled the MRI safety criterions (e.g., no ferromagnetic object in body, not being pregnant). The study was approved by the regional ethical committee and prior to participation, all participants gave written informed consent according to the Declaration of Helsinki. Participants received gift cards for study completion.

\section{Magnetic Resonance Imaging Data Acquisition Protocol}

The MRI data acquisition was conducted on a whole-body 3T clinical MRI scanner at Karolinska hospital in Huddinge (Magnetom Prisma-fit, Siemens Medical Solutions, Erlangen, Germany) equipped with a 32-channel phased-array receiving head coil. The 3D T1-weighted MPRAGE images were acquired using the following main acquisition parameters: $\mathrm{TE} / \mathrm{TR}=2.52 / 1900 \mathrm{~ms}$, flip angle $=9^{\circ}, 1 \mathrm{~mm}$ isotropic voxel size with matrix size $=256 \times 256 \times 176$, in-plane generalized autocalibrating partial parallel acquisition (GRPPA) factor $=2$, pixel band width $=170 \mathrm{~Hz}$. Each T1-weighted MRI session lasted 4 min and $23 \mathrm{~s}$.

\section{Image Preprocessing and Quality Control}

The Computational Anatomical Toolbox (CAT12.3) (Gaser and Dahnke 2016), implemented in MATLAB (Mathworks, Natick, MA, USA) was used for the T1-weighted MRI data preprocessing. The CAT12.3 longitudinal segmentation for voxel-based morphometry (VBM) was used and spatial registration to the Montreal Neurological Institute coordinate space was performed on all images using the geodesic shooting algorithm (Ashburner and Friston 2011). Furthermore, a surface-based longitudinal preprocessing pipeline (Reuter et al. 2012) implemented in Freesurfer (v 6.0.1) was also applied in the T1-weighted images.

Before preprocessing, all raw data $(n=52)$ were manually reviewed. Structural data acquired from one individual of those initially recruited was excluded due to excessive movement artifacts (i.e., ringing artifacts on the T1-weighted image). After preprocessing, sample homogeneity check was performed in accordance with the CAT12 manual. Based on the Mahalanobis distance on mean voxel correlations and the weighted average quality measure, four more $(7.8 \%, 4 / 51)$ participants were removed from further analysis. Thus, the final sample included complete data from 47 individuals. The mean weighted overall image quality was $85.9 \pm 0.3 \%$. There was no image quality difference between the conditions (picture vs. fixation cross; $t(46)=0.13, P=0.895)$. The segmented and spatially normalized T1-weighted MRI data were smoothed using a Gaussian kernel with $8 \mathrm{~mm}$ full width at half maximum (FWHM) in statistical parametric mapping (SPM) software version 12.

\section{Statistical Analyses}

Behavioral data were evaluated using STATA (StataCorp LLC. 2017. Stata Statistical Software: release 15.1. College Station, TX, USA). To evaluate the tentative effect of condition (picture vs. fixation cross) on the T1-weighted images smoothed VBM images were implemented in a paired two-tailed t-test in SPM. The analysis was performed with a primary whole-brain search, alpha set at voxel-wise $P<0.001$, and the obtained minimum cluster extent was 304 voxels (family-wise error, FWE, corrected $P<0.05)$. The primary results were obtained using a SPM $F$ contrast including voxel-wise changes in both directions between the conditions ([1 -1; -11$])$, and follow-up one-tailed t-tests were used to determine the direction (increase or decrease) of 
Table 1 Whole-brain voxel-wise comparisons (two-tailed paired t-test; picture vs. fixation cross).

\begin{tabular}{|c|c|c|c|c|c|c|c|}
\hline \multirow[t]{2}{*}{ Brain regions } & \multicolumn{3}{|c|}{ MNI } & \multirow[t]{2}{*}{ Average volume increase } & \multirow[t]{2}{*}{$z$} & \multirow[t]{2}{*}{ Cluster size ( $n$ voxels) } & \multirow[t]{2}{*}{$P$ FWE } \\
\hline & $\mathrm{x}$ & $\mathrm{y}$ & $\mathbf{z}$ & & & & \\
\hline Left visual cortex cluster & & & & $1.41 \%$ & & 304 & 0.038 \\
\hline Lingual gyrus & -18 & -86 & -9 & & 3.88 & & \\
\hline Fusiform gyrus & -24 & -86 & -15 & & 3.81 & & \\
\hline Right visual cortex cluster & & & & $1.33 \%$ & & 560 & 0.002 \\
\hline Lingual gyrus & 12 & -88 & -9 & & 3.73 & & \\
\hline Fusiform gyrus & 33 & -76 & -14 & & 3.91 & & \\
\hline
\end{tabular}

Abbreviations: T1-w, T1-weighted; VBM, voxel-based morphometry; MNI, Montreal Neurological Institute space; FWE, family-wise error. Note: The analysis included 47 participants.
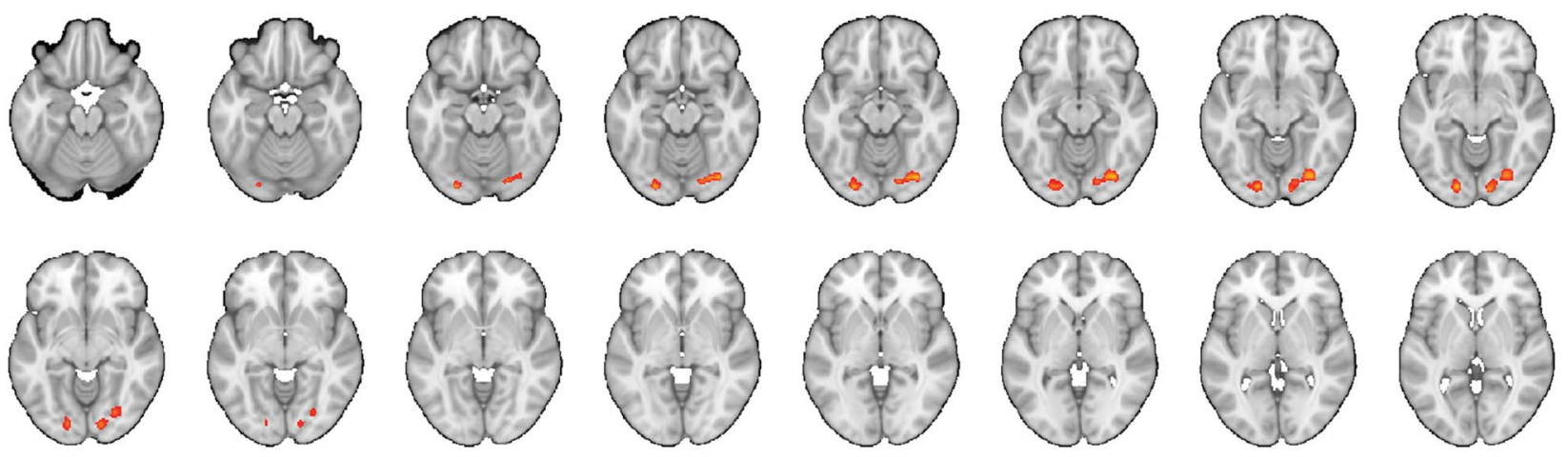

12.50

SPM F contrast (picture vs fixation; threshold $=300$ voxels)

Figure 1. VBM GM volume enlargements while viewing pictures. Whole-brain analysis on VBM GM images demonstrating rapid regional changes ( $\leq 263$ seconds) in bilateral clusters of the occipital lobe, suggesting volumetric increase in the visual cortex while viewing pictures. Whole-brain plot demonstrating significant changes while participants were viewing complex arousing pictures relative to a fixation cross (the figure only include clusters surviving $P<0.001, k$ threshold $>300$ voxels). The color bar represents F-values ranging from 12.5 (red) to 22.5 (yellow). The anatomical underlay is a T1-weigthed image from Keuken et al., 2014 (NeuroImage).

the effect. An explicit mask was entered to restrict the models to only include GM volume across the whole-brain. Significant clusters were extracted (mean voxel value of cluster) and used to calculate the percentage of change between the conditions, and the within-subject Cohen's $d$ effect-size.

In addition to the conventional second-level SPM analysis, two approaches were added. First, a probabilistic threshold-free cluster enhancement (PTFCE) technique was used to reduce possible false positive voxels and "cluster leaking" issues in clusterlevel inferences (Spisák et al. 2019). The pTFCE was implemented in a SPM $F$ contrast and the suggested minimum cluster extent was 623 voxels. Second, a non-parametric permutation testing methodology in the statistical non-parametric mapping (SnMP) software (Nichols and Holmes 2002) was used. VBM images were implemented in a paired t-test in SnMP ( $\mathrm{T}$ contrast evaluating differences between the picture condition and the fixation cross condition). The number of permutations was set at 5000, and the cluster-defining threshold was $t>3.09$, and a cluster-level alpha at 0.05 after FWE correction was considered significant. An explicit mask was entered to restrict the model to only include GM volume across the whole-brain. The critical suprathreshold cluster size was 377 voxels in this model.

For the surface-based cortical thickness measure (Freesurfer preprocessed images smoothed with $10 \mathrm{~mm}$ FWHM kernel), each individual's percentage of change (fixation cross vs. picture) was implemented in one sample t-test to determine change in thickness in the left and right hemispheres. False discovery rate was set at 0.05 to correct for multiple comparisons with Monte Carlo simulation. Clusters surviving $P<0.001$ at an uncorrected level are also reported. In addition, permutation simulation was performed to apply a cluster-wise correction for multiple comparisons (Greve and Fischl 2018) and reduce the false positive rate. Vertex-wise/cluster-forming threshold was set at $3(P<0.001)$, the number of iterations was 1000 , and the cluster-wise alpha 0.05 .

\section{Data Availability}

All data in the manuscript and the supplementary materials is available upon request.

\section{Results}

Within-subject voxel-wise whole-brain analysis on VBM (Gaser and Dahnke 2016) GM images, found regional and bilateral clusters in the occipital lobe, suggesting that GM volume change in the visual cortex while viewing pictures, see Table 1 and Figure 1 for details. A follow-up one-tailed t-test confirmed the direction, that is, increased volume while viewing pictures. A majority of the participants showed an actual increase (above $0 \%$ ) in left 


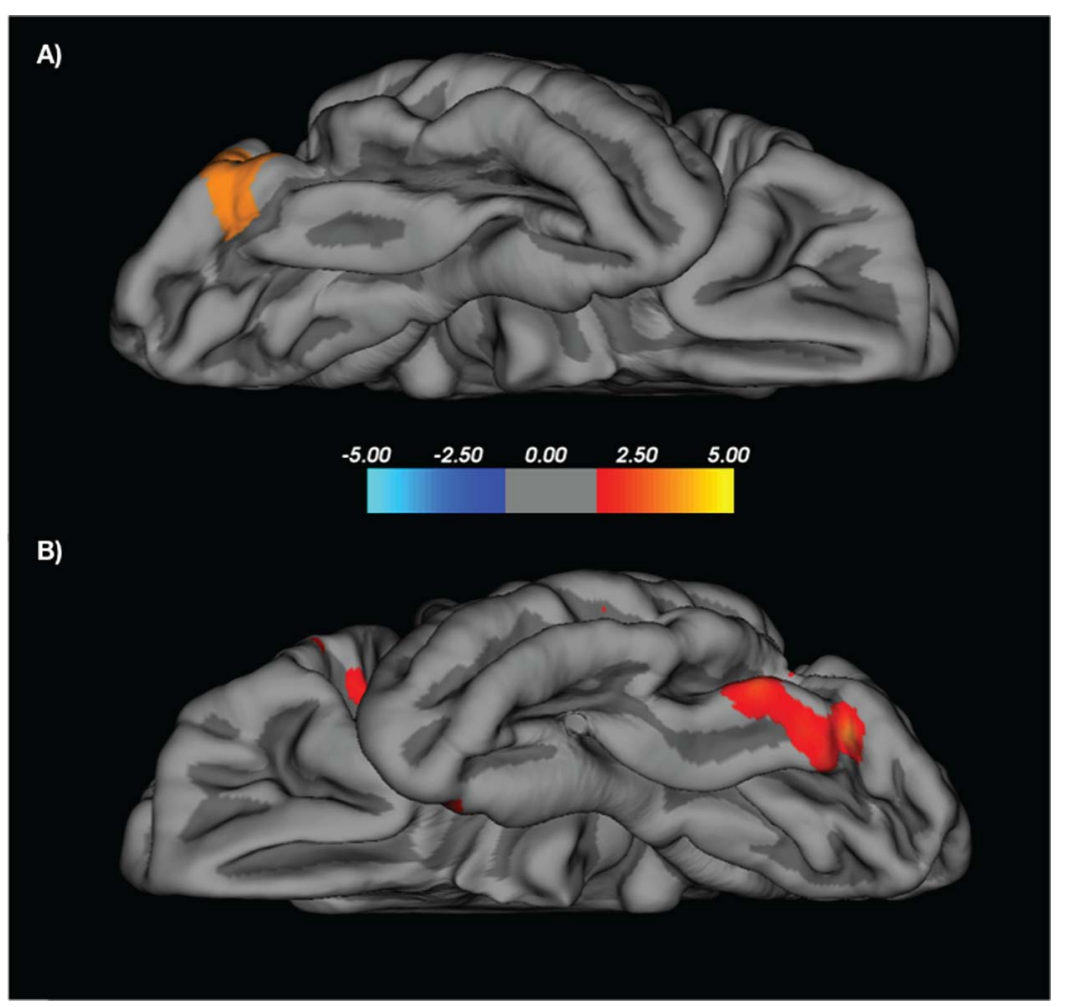

Figure 2. Surface-based cortical thickness enlargements while viewing pictures. Whole-brain analysis on surface-based cortical thickness demonstrating percentage of change in (A) left occipital lobe (logarithmic $P=3.44,378 \mathrm{~mm}^{2}$, uncorrected $P<0.001$ ), and (B) right occipital lobe (logarithmic $P=2.92,859 \mathrm{~mm}{ }^{2}, P<0.05$ after Monte Carlo simulation) suggesting increased thickness in the visual cortex while viewing pictures. Whole-brain plot demonstrating significant change while participants viewed complex arousing pictures relative to a fixation cross. The scale is logarithmic and shows values [-log10(P-value)] with red-yellow color indicating enlargements in cortical thickness.

(78.7\%, 37/47) and right $(70.2 \%, 33 / 47)$ visual cortex GM volume. The within-subject Cohen's $d$ effect sizes were small: left cluster: $d=0.10$ (95\% CI 0.06, 0.15), right cluster: $d=0.12$ (95\% CI 0.07, 0.17).

By use of pTFCE, as well as a non-parametric permutation testing in SnMP, both approaches provide additional support for the notion that GM volume in the left and right visual cortex increase rapidly. See the Supplementary Material for details.

Estimations on the segmented brain tissues were total

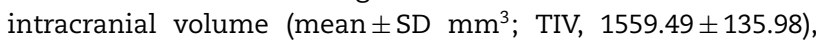
GM (691.62 \pm 58.7$)$, white matter (WM, 527.2 \pm 55.8$)$, and cerebrospinal fluid (CSF, $340.7 \pm 50.1$ ). There were no significant differences between the conditions (pictures vs. fixation cross) on TIV $(t(46)=1.34, P<0.187)$, GM $(t(46)=0.442, P=0.660)$, or $\mathrm{WM}$ $(\mathrm{t}(46)=1.21, P=0.232)$, but participants differed in CSF across the two conditions $(t(46)=2.05, P=0.046)$, suggesting less global CSF while viewing pictures relative to a fixation cross. The main result on GM alterations (i.e., picture vs. fixation cross) remained unchanged when adding global CSF as a nuisance variable.

In addition to VBM, by use of a surfaces-based method implemented in Freesurfer (Dale et al. 1999) to measure cortical thickness, increased occipital lobe thickness while viewing pictures was also found. Right lateral occipital survived correction for multiple comparisons ( $\log 10$ ( $P$-value $)=2.92$, clusterwise $P=0.0005,859 \mathrm{~mm}^{2}$ surface area, MNI xyz [43, $-78,-3]$. Although not significant after correction, left lateral occipital cortex was the only cluster surviving at an uncorrected $(P<0.001)$ level $(-\log 10$ ( $P$-value $)=3.44,378 \mathrm{~mm}^{2}$ surface area, MNI xyz[-35, $-81,-21])$. See also Figure 2 for details. After cluster-wise correction for multiple comparisons, clusters did however not survive.

\section{Discussion}

Forty-seven healthy individuals were assessed while being scanned with MRI under two conditions (randomized to start with either viewing complex arousing pictures or a fixation cross). The study provides evidence of rapid morphological changes in less than $263 \mathrm{~s}$ in the human "visual cortex" (i.e., lingual and fusiform gyrus of the occipital cortex) while passively viewing of complex arousing pictures. This was demonstrated using both VBM and a surface-based cortical thickness preprocessing technique, as well as univariate and multivariate analytics.

Previous studies that used noninvasive neuroimaging in humans and non-human primates have suggested GM volume changes within hours (the T1-weighted anatomical measurements were separated by approximately $2 \mathrm{~h}$ ). For example, 7 individuals had pharmacotherapy with the dopamine D2 antagonist haloperidol (Tost et al. 2010), and 10 rhesus monkeys were exposed to antiepileptic drugs (Tang et al. 2015). Further, enlargement in the visual cortex GM have been shown after less than 2 hours of training in a color naming learning task, still, the brain measurements were separated by 3 days (Kwok et al. 2011). Evidence from these studies indicate that brain morphology alters in response to both internal and external 
environmental influences, within days or hours of exposure. We add that morphological changes occur and can be detected already while being scanned.

If the current findings represent dynamics in the brain's GM volume and cortical thickness, what specifics in morphology change that fast? Experimental studies based on animal research offers some insights on rapid brain morphology change. Dendritic spine plasticity is one likely target, for a review see Alvarez and Sabatini (2007). For example, visual cortex spine plasticity in response to altered sensory inputs has been demonstrated in an adult mouse model (Keck et al. 2008). With advanced optogenetics, evidence for structural plasticity of dendritic spines has also been detected in a short time window of less than $2 \mathrm{~s}$ (Yagishita et al. 2014). However, it still remains unclear what brain volume/thickness derived from T1-weighted images truly reflects, for a review see Tardif et al (Tardif et al. 2016). Neuroplasticity studies (Dayan and Cohen 2011; Lövdén et al. 2013) suggest that the macroscopic assessment of GM volume with T1-weighted imaging represent a complex mixture of different cellular mechanisms, for example, plasticity in synapses, neurons, and glial cells (Wenger et al. 2017a).

Another possible explanation is that changes in brain perfusion can also lead to local variations in $\mathrm{T} 1$ and, therefore, signal intensity changes in T1-weighted images. It has been shown that the manipulation of brain oxygenation level by hyperoxia challenge (Haddock et al. 2013) and maneuver of cerebral blood flow with pharmaceutical agents can all lead to changes of T1-weighted images (Franklin et al. 2012; Ge et al. 2017). We shall consider a voxel as a composition of multiple compartments consisting of GM tissue, vasculature, extracellular space, and CSF. In principle, the amplitude of the resulting T1-weighted signal is dependent on the relative volume fractions, characteristic T1 value for each compartment, and the water exchange dynamics between the different compartments (Yang et al. 2018). To detect neural plasticity change in the brain tissue with T1-weighted MRI, and dissociate the contributions from other vascular components, we need to implement an MRI protocol that allows concomitant assessment of cerebral blood flow, blood volume, $\mathrm{T} 1$ and $\mathrm{T} 2 *$. There are advanced techniques available. Synthetic T1-weighted images derived from different subsets of MRI parameters can be used to obtain microstructural properties of brain tissue, for example, quantitative MRI (Lorio et al. 2016). As suggested in previous studies using conventional T1-weighted acquisitions (Franklin et al. 2012; Ge et al. 2017), changes in the vasculature can masquerade as changes in GM volume. It is important to remind us this vulnerability of T1-weighted MRI in assessing neural plasticity of the brain tissues. However, neither experimental evidence nor theoretical consideration supports the notion that the observed rapid change in T1-weighted images reflects solely physiological changes in the vasculature. In a recent study of short-term VBM change associated with sensory stimuli (Ge et al. 2017), it was shown that significant VBM alteration remains even after correcting the contribution from cerebral blood flow augmentation. This is also supported by the fact that brain regions with VBM change overlap only partially with the results from blood oxygen level-dependent (BOLD)- functional magnetic resonance imaging (fMRI) or cerebral blood flow measurements (Franklin et al. 2012). The non-overlapping regions are typically outside the visual system (Ge et al. 2017), unlike the excellent overlap between BOLD and cerebral blood flow results (Mayhew et al. 2014). Furthermore, we should point out that the TE (2.5 ms) parameter used for T1-weighted MRI is quite short compared with the T2* values of the brain tissues, therefore, T1-weighted MRI is insensitive to BOLD contrast. It is also well known that T1 remains nearly constant in a wide range of blood oxygenation (Thulborn 2012).

Rapid change in morphology can only to some degree be sensitive to the brain's current actions or blood flow, otherwise T1-weighted derived brain anatomy would not yield such accurate predictions of chronological age (i.e., \pm 5 years) (Franke et al. 2010; Ball et al. 2017). Hence, brain morphology appears to be both dynamic and stable over time. The rapid dynamic feature of volume and thickness, as found in the current study, also suggests that T1-weighted images, to some degree, may be affected by the individual's current mental state (e.g., thoughts and feelings). Because all participants underwent two image acquisitions with visual conditions of different complexity, and the order of presentation was randomized across participants, carry-over effects from one condition to the other are not likely. The current study design and results suggest that the morphological change was bidirectional, that is, both volume/thickness increases (fixation $<$ pictures) and reductions (pictures $>$ fixation). If brain volume and thickness are affected by mental state, it is possible that previous experimental tasks can affect the measure of brain morphology, similar to what has been shown to impact resting-state BOLD-fMRI (Tung et al. 2013). However, this research question goes beyond the current study. Nonetheless, it is common to offer participants music, radio, or even movies while acquiring anatomical images. Such conditions could potentially impact measures of GM volume and cortical thickness. This is certainly an issue that also needs to be considered in multi-site studies, for example, consortiums with structural brain images where the procedure for acquiring the MRI data may vary across sites.

It is possible that issues related to image registration and head motion could confound the current results. This seems particularly important because participants may have moved more while viewing attention-capturing arousing pictures, as compared to a stable fixation cross. However, we carefully checked all raw structural images and excluded five individuals due to heavy motion, that is, ringing artifacts or lower quality, as indicated in the image preprocessing steps. Moreover, no difference in image quality between the conditions was found. Reuter and colleagues reported that GM volume estimates could be biased by head motion, and showed that motion appears as loss of volume (Reuter et al. 2015). In contrast, the current study only found enlargement, and not reductions, of occipital GM volume while viewing pictures. Further, effects related to motion are expected to appear by the edges of brain tissue, which was not evident in the current study.

This study was randomized and counterbalanced, all individuals were assessed at multiple time points using a withinsubject design and stimuli selected to maximize the difference in visual complexity. There are different techniques to preprocess structural T1-weighted images, and several statistical approaches available. By use of VBM, rapid increase of visual cortex GM volume is reported, and the result was stable using both parametric and non-parametric cluster-level correction techniques. By use of a surface-based method to assess cortical thickness, there was support for the notion of rapid visual cortex enlargement, but only after applying the conventional Monte Carlo simulation correction.

To our knowledge this is the first morphological study in humans to directly assess rapid changes in GM volume and cortical thickness during an ordinary T1-weighted scanning 
session. Importantly, we found that indices of brain morphology changed rapidly in $263 \mathrm{~s}$, suggesting that the interpretation of GM volume and cortical thickness as morphometric trait measures needs to be revised.

\section{Supplementary Material}

Supplementary material is available at Cerebral Cortex online.

\section{Funding}

Marcus and Amalia Wallenberg Foundation.

\section{Notes}

We are grateful to Natalie Ebner and Tomas Furmark for providing valuable comments on the current manuscript. Funding bodies had no role in the study setup, data interpretation, or reporting. Conflict of Interest. None declared.

\section{Authors contributions}

Funding acquisition: SH and HF. Conceptualization: KNTM, HF and SH. Project administration: DSC and KNTM. Methodology, formal analysis, and software: KNTM, AM, and TQL. KNTM wrote the original draft, and HF, SH, TQL, DSC, and AM were involved in reviewing and editing. All authors read and approved the final manuscript.

\section{References}

Alvarez VA, Sabatini BL. 2007. Anatomical and physiological plasticity of dendritic spines. Annu Reu Neurosci. 30:79-97.

Ashburner J, Friston KJ. 2011. Diffeomorphic registration using geodesic shooting and Gauss-Newton optimisation. Neuroimage. 55:954-967.

Ball G, Adamson C, Beare R, Seal ML. 2017. Modelling neuroanatomical variation during childhood and adolescence with neighbourhood-preserving embedding. Sci Rep. 7: 17796.

Benzinger TLS, Blazey T, Jack CR Jr, Koeppe RA, Su Y, Xiong C, Raichle ME, Snyder AZ, Ances BM, Bateman RJ et al. 2013. Regional variability of imaging biomarkers in autosomal dominant Alzheimer's disease. Proc Natl Acad Sci U S A. 110:E4502-E4509.

Dale AM, Fischl B, Sereno MI. 1999. Cortical surface-based analysis. I. Segmentation and surface reconstruction. Neuroimage. 9:179-194.

Dayan E, Cohen LG. 2011. Neuroplasticity subserving motor skill learning. Neuron. 72:443-454.

Draganski B, Gaser C, Busch V, Schuierer G, Bogdahn U, May A. 2004. Neuroplasticity: changes in grey matter induced by training. Nature. 427:311-312.

Erickson KI, Voss MW, Prakash RS, Basak C, Szabo A, Chaddock L, Kim JS, Heo S, Alves H, White SM et al. 2011. Exercise training increases size of hippocampus and improves memory. Proc Natl Acad Sci U S A. 108:3017-3022.

Fischer H, Wright CI, Whalen PJ, McInerney SC, Shin LM, Rauch SL. 2003. Brain habituation during repeated exposure to fearful and neutral faces: a functional MRI study. Brain Res Bull. 59:387-392.

Franke K, Ziegler G, Klöppel S, Gaser C, Initiative A's DN. 2010. Estimating the age of healthy subjects from T1-weighted
MRI scans using kernel methods: exploring the influence of various parameters. Neuroimage. 50:883-892.

Franklin TR, Wang Z, Shin J, Jagannathan K, Suh JJ, Detre JA, O'Brien CP, Childress AR. 2012. A VBM study demonstrating "apparent" effects of a single dose of medication on T1weighted MRIs. Brain Struct Funct. 218:97-104.

Gaser C, Dahnke R. 2016. CAT-a computational anatomy toolbox for the analysis of structural MRI data. HBM. 2016: 336-348.

Ge Q, Peng W, Zhang J, Weng X, Zhang Y, Liu T, Zang Y-F, Wang Z. 2017. Short-term apparent brain tissue changes are contributed by cerebral blood flow alterations. PLoS One. 12:e0182182.

Greve DN, Fischl B. 2018. False positive rates in surface-based anatomical analysis. Neuroimage. 171:6-14.

Haddock B, Larsson HBW, Hansen AE, Rostrup E. 2013. Measurement of brain oxygenation changes using dynamic $\mathrm{T}(1)$ weighted imaging. Neuroimage. 78:7-15.

Hölzel BK, Carmody J, Vangel M, Congleton C, Yerramsetti SM, Gard T, Lazar SW. 2011. Mindfulness practice leads to increases in regional brain gray matter density. Psychiatry Res. 191:36-43.

Hoogman M, Bralten J, Hibar DP, Mennes M, Zwiers MP, Schweren LSJ, van Hulzen KJE, Medland SE, Shumskaya E, Jahanshad $\mathrm{N}$ et al. 2017. Subcortical brain volume differences in participants with attention deficit hyperactivity disorder in children and adults: a cross-sectional mega-analysis. Lancet Psychiatry. 4:310-319.

Keck T, Mrsic-Flogel TD, Vaz Afonso M, Eysel UT, Bonhoeffer T, Hübener M. 2008. Massive restructuring of neuronal circuits during functional reorganization of adult visual cortex. Nat Neurosci. 11:1162-1167.

Keuken MC, Bazin P-L, Crown L, Hootsmans J, Laufer A, MüllerAxt C, Forstmann BU. 2014. Quantifying inter-individual anatomical variability in the subcortex using $7 \mathrm{~T}$ structural MRI. NeuroImage. 94:40-46.

Kwok V, Niu Z, Kay P, Zhou K, Mo L, Jin Z, So K-F, Tan LH. 2011. Learning new color names produces rapid increase in gray matter in the intact adult human cortex. Proc Natl Acad Sci U S A. 108:6686-6688.

Lang PJ. 2005. International affective picture system (IAPS): affective ratings of pictures and instruction manual. Technical Report A-6.

Lorio S, Kherif F, Ruef A, Melie-Garcia L, Frackowiak R, Ashburner J, Helms G, Lutti A, Draganski B. 2016. Neurobiological origin of spurious brain morphological changes: a quantitative MRI study. Hum Brain Mapp. 37:1801-1815.

Lövdén $\mathrm{M}$, Wenger $\mathrm{E}$, Mårtensson J, Lindenberger U, Bäckman L. 2013. Structural brain plasticity in adult learning and development. Neurosci Biobehav Rev. 37:2296-2310.

Maguire EA, Gadian DG, Johnsrude IS, Good CD, Ashburner J, Frackowiak RS, Frith CD. 2000. Navigation-related structural change in the hippocampi of taxi drivers. Proc Natl Acad Sci U S A. 97:4398-4403.

Mayhew SD, Mullinger KJ, Bagshaw AP, Bowtell R, Francis ST. 2014. Investigating intrinsic connectivity networks using simultaneous BOLD and CBF measurements. Neuroimage. 99:111-121.

Nichols TE, Holmes AP. 2002. Nonparametric permutation tests for functional neuroimaging: a primer with examples. Hum Brain Mapp. 15:1-25.

Nyberg L, Salami A, Andersson M, Eriksson J, Kalpouzos G, Kauppi K, Lind J, Pudas S, Persson J, Nilsson L-G. 2010. 
Longitudinal evidence for diminished frontal cortex function in aging. Proc Natl Acad Sci U S A. 107:22682-22686.

Reuter M, Schmansky NJ, Rosas HD, Fischl B. 2012. Withinsubject template estimation for unbiased longitudinal image analysis. Neuroimage. 61:1402-1418.

Reuter M, Tisdall MD, Qureshi A, Buckner RL, van der Kouwe AJW, Fischl B. 2015. Head motion during MRI acquisition reduces gray matter volume and thickness estimates. Neuroimage. 107:107-115.

Spisák T, Spisák Z, Zunhammer M, Bingel U, Smith S, Nichols T, Kincses T. 2019. Probabilistic TFCE: a generalized combination of cluster size and voxel intensity to increase statistical power. Neuroimage. 185:12-26.

Tang Y, Yu X, Zhang X, Xia W, Wu X, Zou X, Li H, Huang X, Stefan $H$, Chen $Q$ et al. 2015. Single-dose intravenous administration of antiepileptic drugs induces rapid and reversible remodeling in the brain: evidence from a voxel-based morphometry evaluation of valproate and levetiracetam in rhesus monkeys. Neuroscience. 303:595-603.

Tardif CL, Gauthier CJ, Steele CJ, Bazin P-L, Schäfer A, Schaefer A, Turner R, Villringer A. 2016. Advanced MRI techniques to improve our understanding of experience-induced neuroplasticity. Neuroimage. 131:55-72.

Thulborn KR. 2012. My starting point: the discovery of an NMR method for measuring blood oxygenation using the transverse relaxation time of blood water. Neuroimage. 62:589-593.

Tost H, Braus DF, Hakimi S, Ruf M, Vollmert C, Hohn F, MeyerLindenberg A. 2010. Acute D2 receptor blockade induces rapid, reversible remodeling in human cortical-striatal circuits. Nat Neurosci. 13:920-922.

Tung K-C, Uh J, Mao D, Xu F, Xiao G, Lu H. 2013. Alterations in resting functional connectivity due to recent motor task. Neuroimage. 78:316-324.

Wenger E, Brozzoli C, Lindenberger U, Lövdén M. 2017a. Expansion and renormalization of human brain structure during skill acquisition. Trends Cogn Sci. 21:930-939.

Wenger E, Kühn S, Verrel J, Mårtensson J, Bodammer NC, Lindenberger U, Lövdén M. 2017b. Repeated structural imaging reveals nonlinear progression of experience-dependent volume changes in human motor cortex. Cereb Cortex. 27:2911-2925.

Yagishita S, Hayashi-Takagi A, Ellis-Davies GCR, Urakubo H, Ishii S, Kasai H. 2014. A critical time window for dopamine actions on the structural plasticity of dendritic spines. Science. 345:1616-1620.

Yang DM, Huettner JE, Bretthorst GL, Neil JJ, Garbow JR, Ackerman JJH. 2018. Intracellular water preexchange lifetime in neurons and astrocytes. Magn Reson Med. 79:1616-1627. 